\title{
Immunohistochemical Localization of Versican, Link Protein and Hyaluronic Acid Binding Protein in the Human Periodontal Ligament
}

\author{
Tadahiko Utsunomiya' ${ }^{1}$, Aki Otsuka², Rei Sato², Masaru Yamaguchi², Kazutaka Kasai², \\ Hirotsugu Yamamoto ${ }^{3}$, Kayo Kuyama1 \\ ${ }^{1}$ Department of Oral Pathology, Nihon University School of Dentistry at Matsudo, Matsudo, Japan \\ ${ }^{2}$ Department of Orthodontics, Nihon University School of Dentistry at Matsudo, Matsudo, Japan \\ ${ }^{3}$ Nihon University, Tokyo, Japan \\ Email: utsunomiya.tadahiko@nihon-u.ac.jp
}

Received 29 June 2014; revised 15 August 2014; accepted 30 August 2014

Copyright (C) 2014 by authors and Scientific Research Publishing Inc.

This work is licensed under the Creative Commons Attribution International License (CC BY).

http://creativecommons.org/licenses/by/4.0/

(c) (i)

Open Access

\section{Abstract}

The purpose of the present study was to histopathologically and immunohistochemically investigate the distribution of proteoglycans in human periodontal ligament (PDL). Specimens from osteotomy and tooth extraction having healthy PDL were studied. Histologically, PDL consisted of fibrous tissues, involving a compact arrangement area and edematous or myxoid area. Immunohistochemically, versican binding region (12C5), versican link protein (8A4) and biotinylated hyaluronic acid binding protein (B-HABP) were distributed in PDL. In addition, positive immunoreactivity for $12 \mathrm{C5}$ and $8 \mathrm{~A} 4$ was stronger in the compact arrangement area than in the edematous or myxoid area. Reactivity for B-HABP was stronger in the edematous or myxoid area than in the compact fibrous area. These results suggest that versican and link protein are associated with fibrous tissues, whereas hyaluronic acid is related to the formation of edematous and/or myxoid tissue in human PDL. These substances may play a role in periodontal homeostasis by protecting against mechanical stress.

\section{Keywords}

Proteoglycan, Periodontal Ligament, Immunohistochemistry 


\section{Introduction}

In human extracellular matrices, collagen resists tensile force, whereas proteoglycans (PGs) play a role in securing space for tissue [1]-[4]. Versican, which is a non-cartilaginous, large modular PG, binds to hyaluronic acid through hyaluronan-binding protein to form aggregates [1] [2], and these aggregates are stabilized by binding link protein.

Although some immunohistochemical studies of PGs in animal periodontal ligaments have been reported [5]-[8], only a few studies on human periodontal tissues have been reported [9], as it is difficult to obtain healthy human periodontal tissues. Therefore, the role of PGs is not fully understood, and it remains necessary to observe the distribution of PGs in human periodontal ligament (PDL) in order to substantiate the evidence and analysis of experimental studies. Versican and link protein, which is a largest member of of hyaluronan-binding PG, has chondroitin 6-sulphate as the major glycosaminoglycan component and some chondroitin 4-salphate [6]. It plays a role to inhibit cell adhesion and protects cells from mineralization. Hyaluronic acid is a polyanionic polymer composed of repeated diasaccharide units (N-acetylglucosamine and glucuronic acid), and plays various biological and mechanical roles such as resisting compression, filling a large space for embryonic development, and creating a cell-free space into which cells eventually migrate [6].

The purpose of the present study was to histologically and immunohistochemically investigate the expression and distribution of versican, link protein and hyaluronic acid in PDL.

\section{Materials and Methods}

The present study used healthy human PDL without any inflammatory changes and bone destruction. We observed teeth with PDL removed in order to secure the space which moves a tooth during orthodontic therapies and jawbones with teeth in order to excise intraosseous neoplastic lesions by partial psteotomy. Specimens consisted of five cases of partial osteotomy and five cases of expedient tooth extraction for orthodontic treatment. After obtaining informed consent, specimens were immediately fixed in $10 \%$ neutral formalin. After decalcification in 10\% formic-formalin, routine paraffin-embedded blocks were prepared and cut at a thickness of $4 \mu \mathrm{m}$. Hematoxylin-eosin staining (HE) and immunohistochemical staining were performed. Slice specimen of the histological observation is shown in Figure 1(a) \& Figure 1(b).
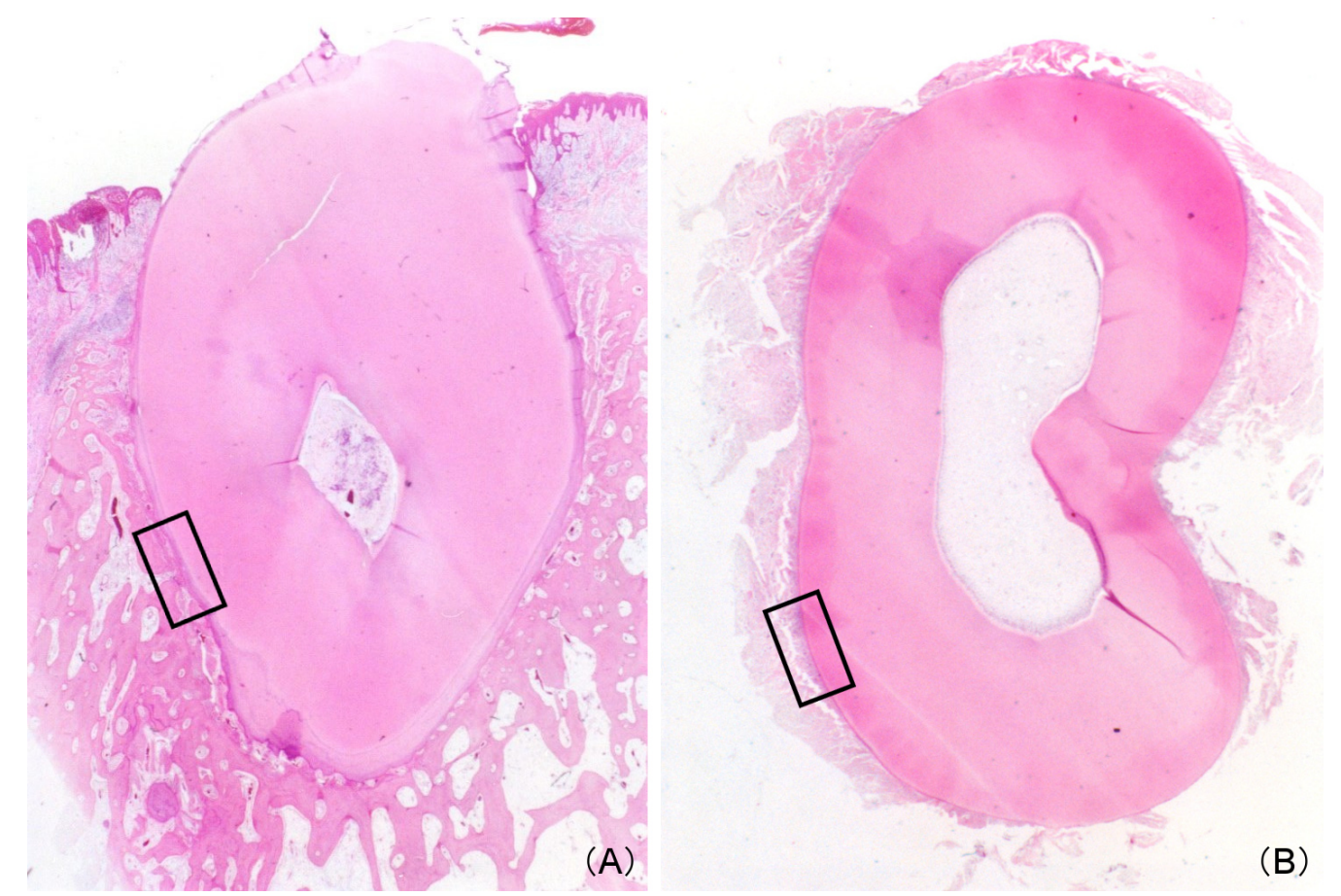

Figure 1. Slice specimen of histological observation. Area of inserted figures is histologically and immunohistochemically observed. (A) Osteotomy specimen; (B) Extracted tooth specimen. 
The envision polymer + kit (DakoCytomation, Glostrup, Denmark) was used for immunohistochemical staining. For pretreatment of staining, sections were digested with chondroitinase ABC (Seikagaku Kogyo, Tokyo, Japan) for 2 hours at $37^{\circ} \mathrm{C}$, and with trypsin (Wako Junyaku, Tokyo, Japan) for 20 minutes at $37^{\circ} \mathrm{C}$. Primary antibodies used in the study were as follows: versican hyaluronan binding region (12C5, monoclonal, dilution of 1:20; Developmental Studies Hybridoma Bank, Iowa City, IA); versican link protein (8A4, monoclonal, dilution of 1:20; Developmental Studies Hybridoma Bank); and biotin-labelled hyaluronic acid binding protein (BHABP; Seikagaku Kogyo, Tokyo, Japan). Sections were visualized by 3,3'-diaminobenzidine and were counterstained with Mayer's hematoxylin.

Dental pulp tissues co-existing in the specimens were used as positive controls. Dental pulp tissues showed positive reactions for primary antibodies used in the present study (built-in-control). As negative controls, sections were incubated with $0.01 \mathrm{M}$ phosphate buffer solution ( $\mathrm{pH}$ 8.6) instead of primary antibodies.

\section{Results}

\subsection{Histological Findings}

PDL mainly consisted of fibroblasts and fibrous tissue with a relatively compact and regular arrangement area of collagenous fibers (compact fibrous area: CF) and edematous or myxoid area (EM) involving collagenous fibers or capillary vessels in both osteotomy and tooth extraction specimens (Figure 1). CF and edematous or myxoid areas were located alternately (Figure 2).

\subsection{Immunohistochemical Findings}

A summary of the immunohistochemical results is shown in Table 1.

Various degrees of positive reactions for 12C5, 8A4 and B-HABP were observed in PDL specimens from osteotomy and tooth extraction. In the osteotomy specimen, the compact fibrous area showed slight-to-moderate positive reactivity for 12C5 (Figure 3(A)) and moderate-to-strong positive reactivity for 8A4 (Figure 3(B)),
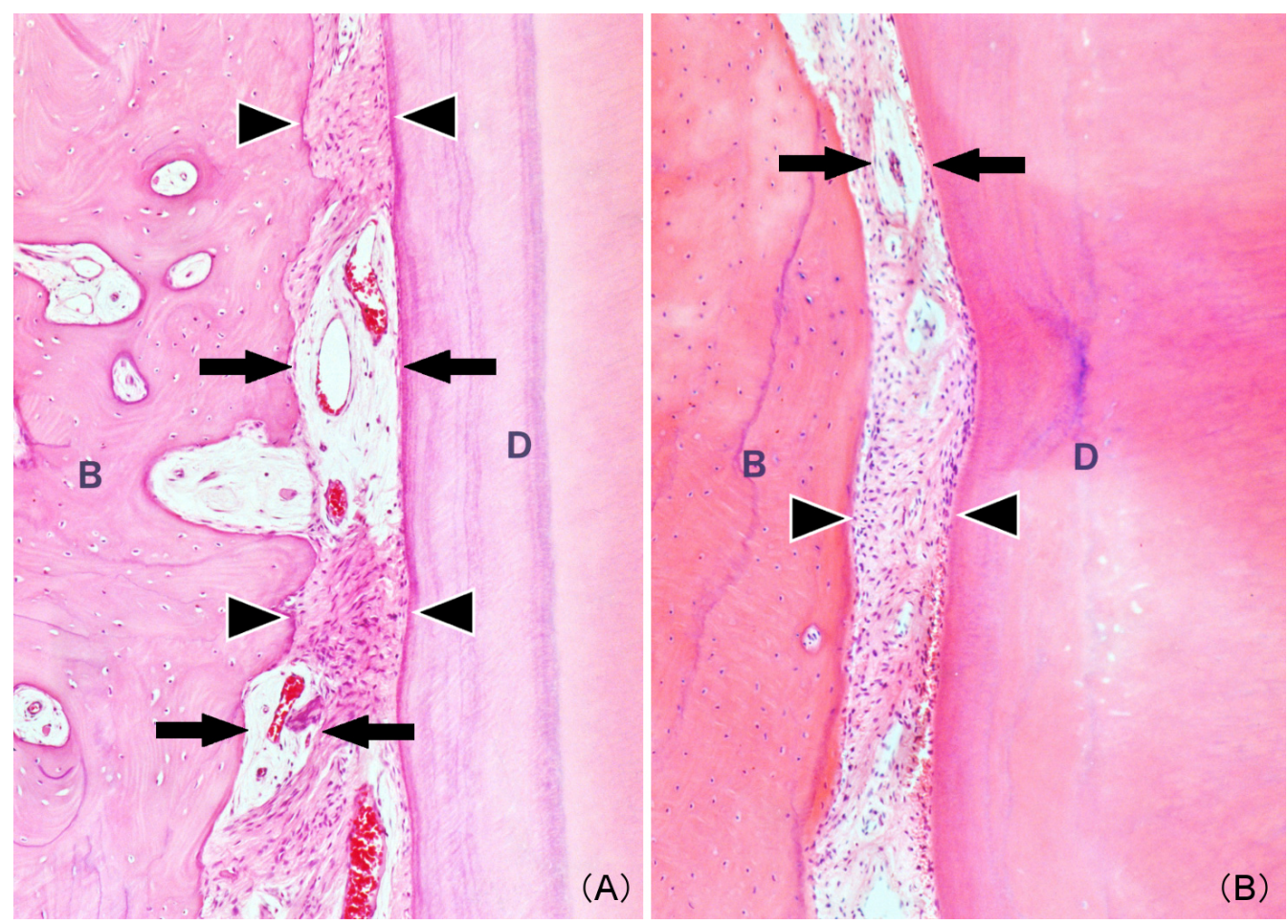

Figure 2. Histological features: Alternately located compact fibrous area (arrowheads) and edematous and/or myxoid area involving blood vessels (arrows) (A) Osteotomy specimen; (B) Extracted tooth specimen (B, alveolar bone; D, dentin) (Magnification 100×). 


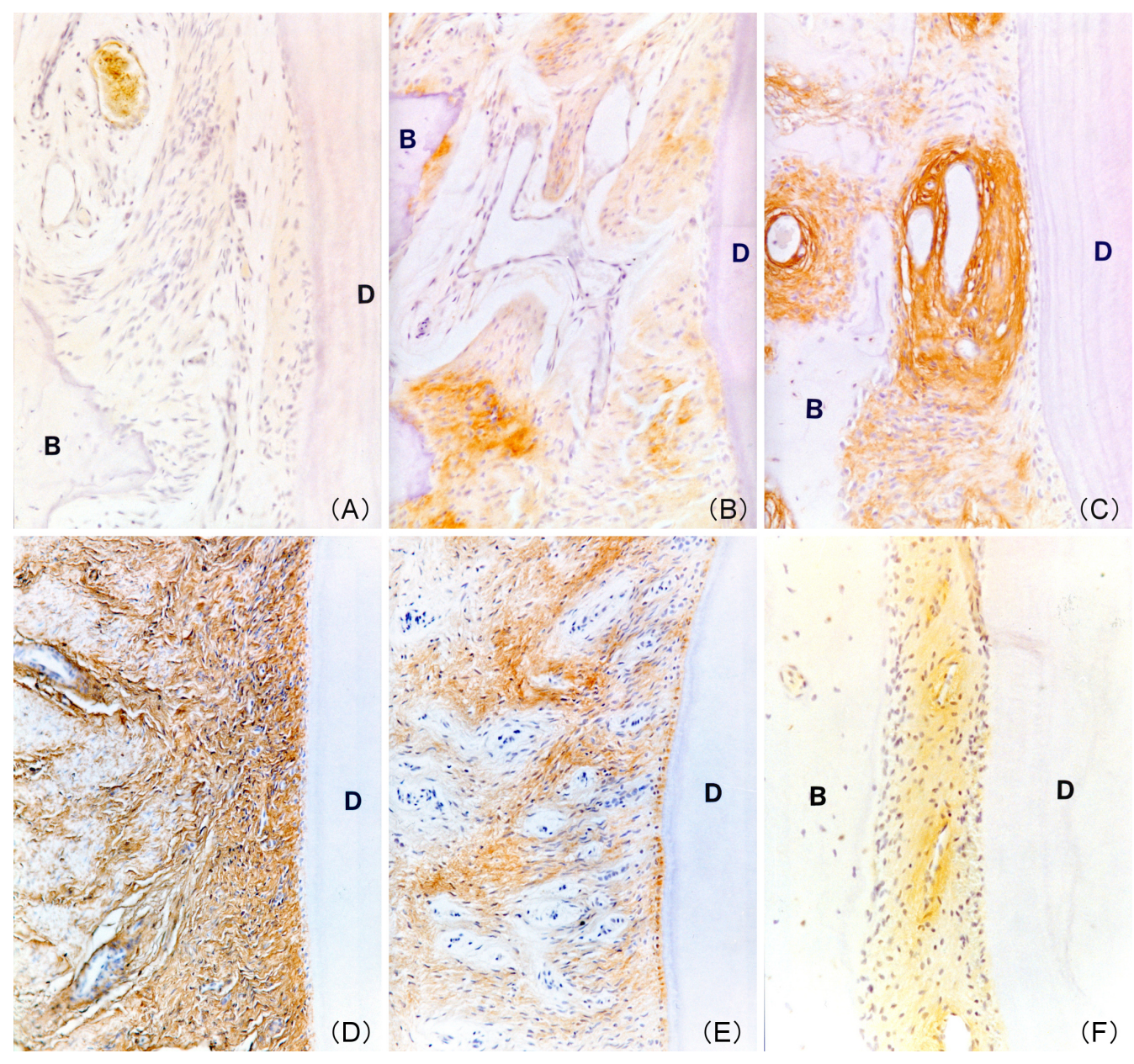

Figure 3. Immunohistochemical features: (A) Slightly positive reactivity for 12C5 in compact fibrous area of osteotomy specimen; (B) Moderately positive reactivity for 8A4 in compact fibrous area of osteotomy specimen; (C) Strongly positive reactivity for B-HABP in edematous and/or myxoid area involving blood vessels of osteotomy specimen; (D) Strongly positive reactivity for 12C5 in compact fibrous area of extracted tooth specimen; (E) Moderately positive reactivity for 8A4 in compact fibrous area of extracted tooth specimen; (F) Moderately positive reactivity for B-HABP in edematous and/or myxoid area involving blood vessels of extracted tooth specimen (B, alveolar bone; $\mathrm{D}$, dentin) (Magnification $200 \times)$.

Table 1. Immunohistochemical results of proteoglycans in human periodontal ligament.

\begin{tabular}{cccccccc}
\hline & \multicolumn{3}{r}{$12 \mathrm{C} 5$} & & & $8 \mathrm{8} 4$ & \multicolumn{3}{c}{ B-HABP } \\
& CF & EM & CF & EM & CF & EM \\
\hline Osteotomy specimen & +-++ & +-- & ++-++ & + & +-++ & ++-++ \\
Extracted tooth & ++-++ & +-- & ++ & ++- & + & & +-++ \\
\hline
\end{tabular}

CF, compact fibrous area; EM, edematous and/or myxoid area; +++, strongly positive; ++, moderately positive; +, slightly positive; -, negative.

while slight-to-moderate positive reactivity for B-HABP (Figure 3(C)) was observed in the matrices. In the tooth extraction specimen, CF also showed moderately or strongly positive reactivity for 12C5 (Figure 3(D)) and 8A4 (Figure 3(E)), whereas slightly positive reactivity for B-HABP (Figure 3(F)) was seen in the matrices. EM showed slightly positive or negative immunoreactivity for B-HABP in the matrices.

In addition, the degree of immunoreactivity in osteotomy specimens was similar to that seen in the tooth extraction specimens, although the osteotomy specimens tended to show stronger activity, except for 12C5 in CF. 


\section{Discussion}

PGs, which are present in all mammalian tissues, are synthesized by most eukaryotic cells [1]. Versican is a large interstitial PG and is generally present in non-cartilaginous connective tissues [1] [4]. Although the mechanical function of PGs is thought to be resilience to compressive force [1], their detailed roles in teeth and/or tooth-associated tissues are not fully understood. PGs, including glycosaminoglycans (GAGs), are believed to be related to the nutritional condition or degeneration of connective tissue [5] [7], overproduction of fibroblasts [10] [11], embryonic conditions [4] or occurrence and/or invasion of neoplasms [10]-[13], but there remains some controversy. Histologically, PDL in the present study showed an alternate arrangement of two compartments (CF and EM), with capillary vessels in accordance with descriptions by other researchers [4]. Generally, the peripheral area of capillary vessels is considered to be the developmental site of undifferentiated mesenchymal cells [6]. Therefore, EM may be associated with embryonic conditions or degenerative changes in the collagenous fibers in PDL.

Imai et al. [5] examined the localization of hyaluronan in rat PDL and suggested that hyaluronan is associated with metabolism. Fujii et al. [6] studied GAGs in the developing PDL of mice and indicated that GAGs may play a special role in the growth and development of periodontal tissues. In addition, Häkkinen et al. [9] observed the distribution of various types of PG in human PDL, and indicated that large molecular weight PGs were intermingled with collagen fibers in deep gingival connective tissues. Alimohamad et al. [14] examined the localization of PGs in human gingiva and concluded that the collagen-binding PGs biglican, fibromodulin and lumican are associated with type I collagen and may collaborate to regulate collagen fibrogenesis, thus suggesting that PGs play an important role in maintaining homeostasis in gingival connective tissues in humans. Sato et al. [15] reported that versican, link protein and hyaluronic acid may form large hydrated aggregates that dissipate the compressive force applied to this tissue during experimental tooth movement. Furthermore, another study reported by Nakamura et al. [16] indicated that woven bone rich in versican changes into lamellar bone containing little versican during experimental bone development, thus suggesting an important role for versican. Recently, gene expressions of PGs in dental pulp and periodontal tissues have been also analyzed suggested that PGs involving versican are closely associated with calcification and inflammation-associated changes [17] [18]. Immunohistochemically, positive reactions for $12 \mathrm{C} 5$ and $8 \mathrm{~A} 4$ in $\mathrm{CF}$ were stronger than in $\mathrm{EM}$, whereas positive reactions for B-HABP in EM were stronger than in CF. The primary antibodies 12C5, 8A4 and B-HABP in the present study showed specificity for versican core protein, link protein and hyaluronic acid, respectively. These results suggest that large molecular weight PGs described by Häkkinen et al. [9] correspond to versican and/or link protein.

The present study showed different degrees of reactivity, as osteotomy specimens tended to show stronger reactivity than tooth extraction specimens, except for 12C5 in CF (Table 1). The cause of these differences was thought to be the conditions of fixation or artifacts due to sampling procedures. The limitation in this presentation is that, influence of rapid mechanical stress on PDL by tooth extraction (extracted tooth specimen) and that of the intraosseous lesion (osteotomy specimen) cannot be denied completely. However, the present study found similar distribution patterns between osteotomy and tooth extraction specimens; therefore, the analyses indicate the ubiquitous nature of PGs in healthy human PDL. These results suggest that hyaluronic acid (hyaluronan) is associated with large amounts of water to form edematous or myxoid tissue, whereas versican core protein and hyaluronan-binding protein (link protein) formed ternary aggregates in accordance with previous studies [1] [2], [7] [8], and these play a role in periodontal homeostasis by protecting against mechanical stress. The results of this study also provide fundamental knowledge regarding the role of PGs in human PDL.

\section{Conclusion}

The present study showed histopathological characteristics and immunohistochemical distribution of proteoglycans in human PDL, and the results suggest that versican and link protein are associated with CF tissues, whereas hyaluronic acid is related to the formation of EM tissue in human PDL. These substances may be associated with periodontal homeostasis by protecting against mechanical stress.

\section{References}

[1] Yanagishita, M. (1993) Function of Prteoglycans in the Extracellular Matrix. Acta Pathologica Japonica, 43, $283-293$.

[2] Heinegard, D. and Oldberg, A. (1993) Connective Tissue and Its Heritable Disorders. Wiley-Liss, New York, $283-209$. 
[3] Reardon, A., Heinegard, D., Mcleod, D., Sheehan, J.K. and Bishop, P.N. (1998) The Large Chondroitin Sulphate Proteoglycan Versican in Mammalian Viterous. Matrix Biology, 17, 325-333.

[4] Sorrell, J.M., Carrino, D.A., Baber, M.A. and Caplan, A.I. (1999) Versican in Human Fetal Skin Development. Anatomy and Embryology, 199, 45-56. http://dx.doi.org/10.1007/s004290050208

[5] Imai, K., Shibutani, T., Horiki, I., Ishihara, T., Kanazawa, A. and Iwayama, Y. (1998) Localization of Hyaluronan in Periodontal Tissues. Journal of Gifu Dental Society, 25, 28-34.

[6] Fujii, T. and Hirabayashi, Y. (1999) Histochemical Studies of Glycosaminoglycans in Developing Periodontal Ligaments of ICR mice. The Anatomical Record, 254, 465-473.

[7] Yamauchi, S., Cheng, H., Neame, P., Caterson, B. and Yamauchi, M. (1997) Identification, Partial Characterization, and Distribution of Versican and Link Protein in Bovine Dental Palp. Journal of Dental Research, 76, 1730-1736. http://dx.doi.org/10.1177/00220345970760110301

[8] Shibata, S., Yoneda, S., Yanagishita, M. and Yamashita, Y. (2000) Isolation of Proteoglycan (Versican) Aggregates from Rat Dental Pulp. Archives of Oral Biology, 45, 563-568.

[9] Häkkinen, L., Oksala, O., Salo, T., Rahemtulla, F. and Larjava, H. (1993) Immunohistochemical Localization of Proteoglycans in Human Periodontium. Journal of Histochemistry and Cytochemistry, 41, 1689-1699.

[10] Shafer, W.G., Hine, M.K., Levy, B.M. and Tomich, C.E. (1983) Textbook of Oral Pathology. 4th Edition, W. B. Saunders Company, Philadelphia, 160-161.

[11] Tomich, C.E. (1974) Oral Focal Mucinosis. A Clinicopathological and Histochemical Study of Eight Cases. Oral Surgery Oral Medicine Oral Pathology, 38, 714-724.

[12] Ozawa, K. and Utsunomiya, T. (2000) A Histochemical and Immunohistochemical Study of Oral Neurofibroma and Schwannoma. With Special Reference to Mucoid Matrix Formation. Nihon University Journal of Oral Science, 26, 156-164.

[13] Zhao, M., Lu, Y., Takata, T., Ogawa, I., Miyauchi, M., Mock, D. and Nikai, H. (1999) Immunohistochemical and Histochemical Characterization of the Mucosubstances of Odontogenic Myxoma. Histogenesis and Differential Diagnosis. Pathology, Research and Practice, 195, 391-397.

[14] Alimohamad, H., Habijanac, T., Larjava, H. and Häkkinen, L. (2005) Colocalization of the Collagen-Binding Proteoglycans Decorin, Biglycan, Fibromodulin and Lumican with Different Cells in Human Gingiva. Journal of Periodontal Research, 40, 73-86.

[15] Sato, R., Yamamoto, H., Kasai, K. and Yamauchi, M. (2002) Distribution Pattern of Versican, Link Protein and Hyaluronic Acid in the Rat Periodontal Ligament During Experimental Tooth Movement. Journal of Periodontal Research, 37, 15-22.

[16] Nakamura, M., Sone, S., Takahashi, I., Mizoguchi, I., Echigo, S. and Sasano, Y. (2005) Expression of Versican and ADAMTS 1, 4, and 5 During Bone Development in the Rat Mandible and Hind Limb. Journal of Histochemistry \& Cytochemistry, 53, 1553-1562.

[17] Okahata, S., Yamamoto, R., Yamakoshi, Y. and Fukae, M. (2011) A Large Chondroitin Sulfate Proteoglycan, Versican, in Porcine Predentin. Journal of Oral Bioscience, 53, 72-81.

[18] Beikler, T., Peters, U., Prior, K., Eisenacher, M. and Flemming T.F. (2008) Gene Expression in Priodontal Tissues Following Treatment. BMC Medical Genimics, 1, 30. 
Scientific Research Publishing (SCIRP) is one of the largest Open Access journal publishers. It is currently publishing more than 200 open access, online, peer-reviewed journals covering a wide range of academic disciplines. SCIRP serves the worldwide academic communities and contributes to the progress and application of science with its publication.

Other selected journals from SCIRP are listed as below. Submit your manuscript to us via either submit@scirp.org or Online Submission Portal.
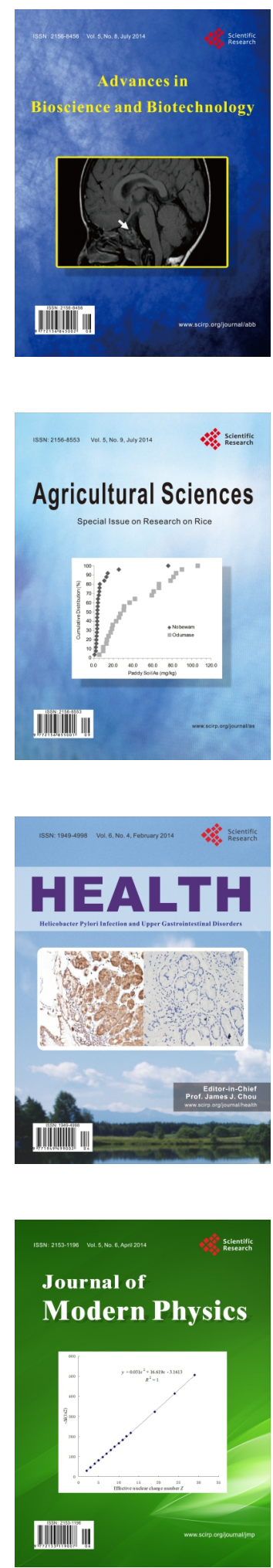
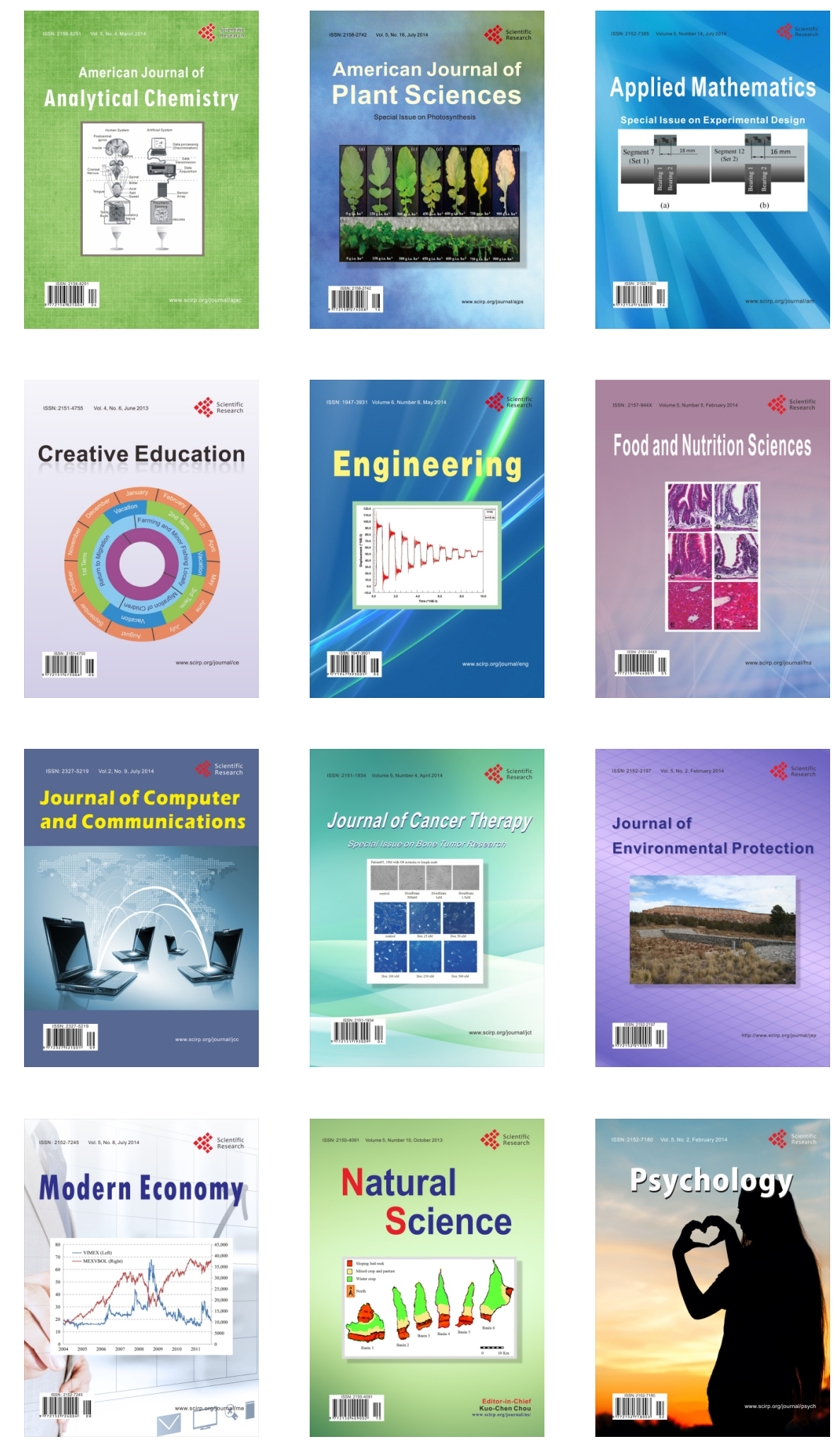